Revista Brasileira de Agricultura Irrigada v.10, nº.4, p. 866 - 874, 2016

ISSN 1982-7679 (On-line)

Fortaleza, CE, INOVAGRI - http://www.inovagri.org.br

DOI: $10.7127 /$ rbai.v10n400462

Protocolo 462.16 - 20/07/2016 Aprovado em 14/08/2016

\title{
IRRIGAÇÃO COM EFLUENTE DE PISCICULTURA NO CULTIVO DE MUDAS DE TOMATE
}

Thiara Silvestre Nascimento ${ }^{1}$, Raimundo Nonato Farias Monteiro ${ }^{2}$, Mariana Alexandre de Lima Sales ${ }^{3}$, Luciane Sperandio Floriano ${ }^{4}$, Alexandre Igor de Azevedo Pereira ${ }^{5}$.

\section{RESUMO}

O aproveitamento de efluentes de viveiros de peixes para a irrigação é um possível complemento de adubação, sendo usado como uma associação rentável tanto econômica como ambiental. O objetivo deste trabalho foi avaliar o desenvolvimento de mudas de tomate, irrigadas com diferentes concentrações de efluente de piscicultura. O trabalho foi realizado Instituto Federal Goiano - Campus de Urutaí - GO. O delineamento estatístico adotado foi inteiramente casualizado com 16 repetições, às quais foram ministradas seis níveis de concentração de água residuária na irrigação. A água de irrigação estava com porcentagem de água residuária 0, 25, 50, 75 e 100\%. As variáveis analisadas foram: altura das plantas e largura das folhas aos $5,10,15,20,25,30,35,40,45$ e 50 dias após a germinação, número de folhas verdadeiras, peso de matéria fresca da parte aérea, peso de matéria fresca do sistema radicular, peso de matéria seca da parte aérea e peso de matéria seca do sistema radicular. Os resultados mostraram que com a elevação das concentrações de água residuária de piscicultura, os valores das variáveis aumentaram, podendo concluir que, a água residuária da piscicultura apresentase como alternativa para reuso do ponto de vista nutricional para as plantas, diminuindo o impacto ambiental negativo dos efluentes desse tipo de criação animal.

Palavras-chave: Lycopersicon esculentum, reuso de água, sistema alternativo.

\section{IRRIGATION WITH EFFLUENT OF PISCICULTURE IN THE CULTIVATION OF TOMATO SEEDLINGS}

\footnotetext{
ABSTRACT

The use of fish ponds of wastewater for irrigation is a possible fertilizer complement, being used as an association profitable both economically and environmentally. The objective of this

${ }^{1}$ Tecnóloga em Irrigação e Drenagem, Mestranda em Agronomia (Irrigação e Drenagem), UNESP/FCA, Botucatu - SP, email: thiarasilvestre@hotmail.com.

2 Tecnólogo em Rec. Hídricos/Irrigação, Dr. em Agronomia (Irrigação e Drenagem), UNESP/FCA, Botucatu - SP.

${ }^{3}$ Tecnóloga em Irrigação e Drenagem, Doutoranda em Agronomia (Irrigação e Drenagem), UNESP/FCA, Botucatu - SP.

${ }^{4}$ Zootecnista, Dra. em Ciência Animal, Profa. IFGoiano, Câmpus de Urutaí - GO.

${ }^{5}$ Eng. Agrônomo, Dr. em Entomologia, Prof. IFGoiano, Câmpus de Urutaí - GO.
} 
study was to evaluate the development of tomato seedlings irrigated with different fish farm effluent concentrations. The work was carried Instituto Federal Goiano - Campus Urutaí - GO. The statistical design was completely randomized with 16 repetitions, which were given six levels of concentration of wastewater for irrigation. Irrigation water was wastewater percentage of $0,25,50,75$ and $100 \%$. The variables analyzed were: plant height and width of the leaves at $5,10,15,20,25,30,35,40,45$ and 50 days after germination, number of true leaves, fresh weight of the aerial part, fresh weight of roots, dry weight of shoot and dry weight of the root system. The results showed that with rising wastewater concentrations of fish, variable values increased, which may conclude that the wastewater from fish farming is presented as an alternative to reuse the nutritional point of view the plants, reducing the environmental impact negative effluent such animal husbandry.

Keywords: Lycopersicon esculentum, water reuse, alternative system.

\section{INTRODUÇÃO}

Os recursos hídricos têm importância fundamental no desenvolvimento de diversas atividades econômicas. Em relação à produção agrícola, a água pode representar até $90 \%$ da constituição física das plantas (SALES, 2014). A falta d'água em períodos de crescimento dos vegetais pode inviabilizar a produção agrícola e até afetar seriamente os ecossistemas equilibrados. Na indústria, por exemplo, para se obter diversos produtos, as quantidades de água necessárias são muitas vezes superiores ao volume gerado pelas estações de tratamento de água (GOMES,2011).

Kummer et al. (2012) afirmam que a utilização de efluentes na agricultura cresceu consideravelmente nos últimos anos em muitos países, inclusive no Brasil; no entanto, ainda não foram suficientemente estudados todos os aspectos positivos e negativos dessa técnica, especialmente sobre as propriedades físicas e químicas do solo, absorção de nutrientes pelas plantas ou sua toxidez. Dentre os principais fatores que vieram a contribuir para que, nos últimos anos, aumentasse o interesse pela irrigação com efluentes, estão a escassez de recursos hídricos, o avanço do conhecimento técnico-científico, a legislação ambiental mais rigorosa e atuante, o maior controle da poluição ambiental, com redução de problemas à saúde humana e animal, a diminuição dos custos de tratamento devido à atuação do solo como forma de disposição e fornecimento de nutrientes e matéria orgânica às plantas, reduzindo os custos com fertilizantes químicos comerciais (SANDRI, 2003).

O uso planejado de águas residuárias implica na necessidade de menor captação dos recursos hídricos primários e de geração reduzida de efluentes, constituindo-se, portanto, em estratégia eficaz para a conservação desse recurso natural, em seus aspectos qualitativos e quantitativos (MEDEIROS et al., 2007), principalmente quando usado na agricultura, servindo como fonte extra de nutrientes, auxiliando no desenvolvimento da cultura (SANDRI et al., 2007).

O aproveitamento planejado de águas residuária na agricultura (área restrita, fácil de confinar e controlar, e altamente eficiente na remoção de poluentes e contaminantes) é uma alternativa para controle da poluição de corpos d'água, disponibilização de água para as culturas, reciclagem de nutrientes e aumento de produção agrícola (MEHNERT, 2003).

A cultura do tomate representa um dos maiores mercados agrícolas brasileiros, ocupando área plantada de 58.000 hectares, dos quais 40.000 hectares são de tomate fresco e 18.000 hectares de tomate destinado ao processamento. A produção alcança aproximadamente três milhões de toneladas por ano, sendo um milhão e 800 mil toneladas de tomate de mesa. A maior parte da produção de tomate concentra-se nos Estados de Goiás, São Paulo e Minas Gerais, totalizando cerca de 65\% da produção nacional (RIBEIRO et al., 2009).

$\mathrm{Na}$ agricultura a sustentabilidade dos sistemas agrícolas é fator primordial, e a integração de vários sistemas implicará no 
melhor aproveitamento de recursos disponíveis em uma propriedade, ocasionando assim, um aporte maior de nutrientes para as plantas. $\mathrm{O}$ aproveitamento de efluentes de viveiros de peixes para a irrigação e possível complemento de adubação, sendo usado como uma associação rentável tanto econômica como ambiental. A utilização de efluentes de viveiros de peixes reduz o custo da água e a quantidade de fertilizantes químicos utilizados (SARIG, 1994).

O objetivo deste trabalho foi avaliar o desenvolvimento de mudas de tomate (Lycopersicon esculentum) cv. Santa Clara 5300, irrigadas com diferentes concentrações de efluente de piscicultura.

\section{MATERIAL E MÉTODOS}

A pesquisa foi conduzida nas dependências do Instituto Federal Goiano Câmpus de Urutaí - GO, no ano de 2010, apresentando latitude de $17^{\circ} 27^{\prime} 52^{\prime \prime}$ Sul e $48^{\circ} 12^{\prime} 13^{\prime \prime}$ Oeste de longitude e com altitude média de $821 \mathrm{~m}$. O clima da região segundo classificação Köppen é caracterizado como Cwa, sendo representado como clima úmido tropical, de inverno seco e verão chuvoso.

As mudas de tomate (Lycopersicon esculentum), cv. Santa Clara 5300 em bandeja com 128 cédulas preenchidas com o substrato Bioplant ${ }^{\circledR}$, que tem como matéria prima casca de Pinus e como agregantes, fibra de coco, casca de arroz e nutrientes, possuindo $\mathrm{pH}$ entre 5,5 - 6,0. O substrato foi peneirado com auxílio de uma peneira com malha de $2 \mathrm{~mm}$ e em seguida colocado na bandeja. Em cada cédula foi inserida uma semente de pimentão, com profundidade de $0,5 \mathrm{~cm}$, e em seguida uma nova camada de substrato foi reposta para a cobertura das sementes, concluindo assim o processo de semeadura.

A água utilizada na irrigação das mudas era oriunda de um poço de decantação, situado no setor de Piscicultura do IFGoiano, Câmpus de Urutaí, e transportada em recipientes para casa de vegetação, de onde eram preparadas as concentrações de 0 (tratamento testemunha), 25, 50, 75, 100 e 125\% da água residuária em água de abastecimento, que seriam aplicadas nas mudas. Foram encaminhas uma amostra de cada concentração para o laboratório de solos, também localizado no IFGoiano, Câmpus de Urutaí para as devidas análises de macro (P, K, $\mathrm{Ca}$ ) e micronutrientes ( $\mathrm{Fe}, \mathrm{Mn}, \mathrm{Cu}, \mathrm{Na}, \mathrm{Cu}, \mathrm{Zn}$,)

$\mathrm{O}$ delineamento experimental foi inteiramente casualizado. As bandejas foram divididas em 6 tratamentos, sendo seis concentrações de água residuária de piscicultura e 16 repetições.

Foram analisadas a altura das plantas (AP) e largura das folhas (LF) aos 5, 10, 15, 20, 25, 30, 35, 40, 45 e 50 dias após a germinação (DAG), com o auxílio de uma trena milimetrada, bem como número de folhas (NF).

Após atingir o tamanho desejado para o transplantio, as mudas de tomate foram avaliadas em peso de matéria fresca da parte aérea (PMFPA) e do sistema radicular (PMFSR), ambas medidas em grama, aferidas com uso de uma balança de precisão de $\pm 0,0001 \mathrm{~g}$. Em seguida foram alocadas em envelopes e colocadas em estufa a $70^{\circ} \mathrm{C}$ por 24 horas para então serem feitas as avaliações de peso de matéria seca da parte aérea (PMSPA) e do sistema radicular (PMSSR).

Os resultados foram submetidos à análise de variância e as médias analisadas pelo teste de Tukey a 5\% de probabilidade, com auxílio do software SISVAR, versão 5.3.

\section{RESULTADOS E DISCUSSÃO}

Os resultados da variável altura de plantas, submetidas a diferentes concentrações de água residuária, podem ser visualizados na Tabela 1. Os dados se mostraram significativamente diferentes aos 15, 25, 30 e 40 dias após a germinação (DAG), tendo as demais avaliações resultados não significativos.

Tabela 1. Altura das plantas (cm) (média $\pm \mathrm{EP}^{1}$ ) de tomate, submetidas a diferentes concentrações de água residuária de piscicultura, até os 50 DAG (dias após a germinação).

\section{Concentrações (\%)}


Nascimento et al.

\begin{tabular}{|c|c|c|c|c|c|c|c|c|c|}
\hline (DAG) & 0 & 25 & 50 & 75 & 100 & 125 & $\mathrm{~F}$ & $P$ & $\mathrm{CV}$ \\
\hline \multirow{2}{*}{5} & $2,14 \pm$ & $2,18 \pm$ & $2,54 \pm$ & $2,15 \pm$ & $2,62 \pm$ & $2,66 \pm$ & \multirow{2}{*}{2,55} & \multirow{2}{*}{0,06} & \multirow{2}{*}{13,10} \\
\hline & $0,06 \mathrm{a}$ & $0,22 \mathrm{a}$ & $0,18 \mathrm{a}$ & $0,09 \mathrm{a}$ & 0,09 a & 0,19 a & & & \\
\hline \multirow{2}{*}{10} & $2,70 \pm$ & $3,00 \pm$ & $2,98 \pm$ & $2,80 \pm$ & $3,23 \pm$ & $3,16 \pm$ & \multirow{2}{*}{2,07} & \multirow{2}{*}{0,11} & \multirow{2}{*}{9,47} \\
\hline & $0,07 \mathrm{a}$ & 0,06 a & 0,18 a & $0,11 \mathrm{a}$ & 0,15 a & 0,19 a & & & \\
\hline \multirow{2}{*}{15} & $3,22 \pm$ & $4,21 \pm$ & $3,92 \pm$ & $3,91 \pm$ & $4,38 \pm$ & $4,28 \pm$ & \multirow{2}{*}{4,75} & \multirow{2}{*}{0,00} & \multirow{2}{*}{9,72} \\
\hline & $0,20 \mathrm{~b}$ & 0,07 a & $0,30 \mathrm{ab}$ & $0,10 \mathrm{ab}$ & 0,10 a & $0,24 \mathrm{a}$ & & & \\
\hline \multirow{2}{*}{20} & $4,29 \pm$ & $5,45 \pm$ & 4,93 \pm & $5,25 \pm$ & $5,38 \pm$ & $5,25 \pm$ & \multirow{2}{*}{2,57} & \multirow{2}{*}{0,06} & \multirow{2}{*}{10,56} \\
\hline & $0,21 \mathrm{a}$ & 0,36 a & $0,37 \mathrm{a}$ & 0,15 a & 0,13 a & $0,27 \mathrm{a}$ & & & \\
\hline \multirow{2}{*}{25} & $4,78 \pm$ & $6,61 \pm$ & $5,77 \pm$ & $6,45 \pm$ & $6,36 \pm$ & $6,50 \pm$ & \multirow{2}{*}{4,54} & \multirow{2}{*}{0,00} & \multirow{2}{*}{10,84} \\
\hline & $0,19 \mathrm{~b}$ & $0,34 \mathrm{a}$ & $0,62 \mathrm{ab}$ & 0,20 a & 0,09 a & 0,23 a & & & \\
\hline \multirow{2}{*}{30} & $5,42 \pm$ & $7,56 \pm$ & $6,80 \pm$ & $6,76 \pm$ & $7,23 \pm$ & $7,79 \pm$ & \multirow{2}{*}{7,26} & \multirow{2}{*}{0,00} & \multirow{2}{*}{9,01} \\
\hline & $0,22 \mathrm{~b}$ & $0,54 \mathrm{a}$ & $0,36 \mathrm{ab}$ & $0,25 \mathrm{ab}$ & 0,17 a & $0,11 \mathrm{a}$ & & & \\
\hline \multirow{2}{*}{35} & $5,78 \pm$ & $8,77 \pm$ & $7,05 \pm$ & $7,40 \pm$ & $8,11 \pm$ & $9,19 \pm$ & \multirow{2}{*}{7,51} & \multirow{2}{*}{0,00} & \multirow{2}{*}{11,77} \\
\hline & $0,36 \mathrm{c}$ & $0,71 \mathrm{ab}$ & 0,48 bc & $0,30 \mathrm{abc}$ & $0,40 a b$ & $0,31 \mathrm{a}$ & & & \\
\hline \multirow[t]{2}{*}{40} & $6,32 \pm$ & $8,70 \pm$ & 7,61 \pm & 7,56 \pm & $8,45 \pm$ & $9,28 \pm$ & \multirow{2}{*}{5,66} & \multirow{2}{*}{0,00} & \multirow{2}{*}{11,01} \\
\hline & $0,25 \mathrm{~b}$ & 0,75 a & $0,36 \mathrm{ab}$ & $0,06 \mathrm{ab}$ & $0,52 \mathrm{a}$ & $0,34 \mathrm{a}$ & & & \\
\hline 45 & $\begin{array}{l}6,56 \pm \\
0,39 \mathrm{c}\end{array}$ & $\begin{array}{c}8,80 \pm \\
0,69 \mathrm{ab}\end{array}$ & $\begin{array}{c}7,54 \pm \\
0,42 \text { bc }\end{array}$ & $\begin{array}{c}7,75 \pm \\
0,18 \mathrm{bc}\end{array}$ & $\begin{array}{c}8,68 \pm \\
0,52 \mathrm{ab}\end{array}$ & $\begin{array}{c}10,10 \pm \\
0,39 \mathrm{a}\end{array}$ & 7,15 & 0,00 & 11,19 \\
\hline 50 & $\begin{array}{l}6,55 \pm \\
0,30 \mathrm{c}\end{array}$ & $\begin{array}{c}8,98 \pm \\
0,64 \mathrm{ab}\end{array}$ & $\begin{array}{c}7,91 \pm \\
0,34 \text { bc }\end{array}$ & $\begin{array}{c}8,18 \pm \\
0,36 \mathrm{bc}\end{array}$ & $\begin{array}{c}8,73 \pm \\
0,55 \mathrm{ab}\end{array}$ & $\begin{array}{c}10,55 \pm \\
0,38 \mathrm{a}\end{array}$ & 8,63 & 0,00 & 10,58 \\
\hline
\end{tabular}

${ }^{1}$ Médias seguidas pela mesma letra, dentro de cada linha, não diferem entre si ao nível de 5\% de probabilidade pelo teste Tukey.

Aos 15 DAG a maior altura das mudas foi observada nas concentrações de $25(4,21 \mathrm{~cm})$, $100(4,38 \mathrm{~cm})$ e $125 \%(4,28 \mathrm{~cm})$, enquanto que as mudas de tomate, sem tratamento com água residuária, obtiveram altura média de $3,22 \mathrm{~cm}$. As mudas de tomate não tratadas com água residuária (0\%), aos 25 , 30 e 40 DAG, também atingiram menores alturas em comparação com os demais tratamentos. Nascimento et al. (2016) estudando a variável altura de plantas para a cultura do pimentão em diferentes concentrações de água residuária de piscicultura, encontraram resultados similares aos dessa pesquisa. De acordo com trabalhos realizados por Augusto et al. (2003), Hussar et al. (2005) e Sanches et al. (2007), a utilização de água residuária como alternativa de água e nutrientes apresentaram resultados semelhantes com bom crescimento das plantas.

A variável AP seguiu comportamento cúbico de regressão $(\mathrm{F}=45,51 ; P=0,00)$, como mostra a Figura 1. A taxa de crescimento das mudas de tomate submetidas a $125 \%$ de água residuária foram 54\% superiores em comparação com aquelas submetidas a $0 \%$. A utilização de efluente tratado na irrigação possibilita melhor crescimento das plantas quando comparado com adubação convencional, devido à existência de nutrientes essenciais para o desenvolvimento das plantas (SOUSA et al., 2006).

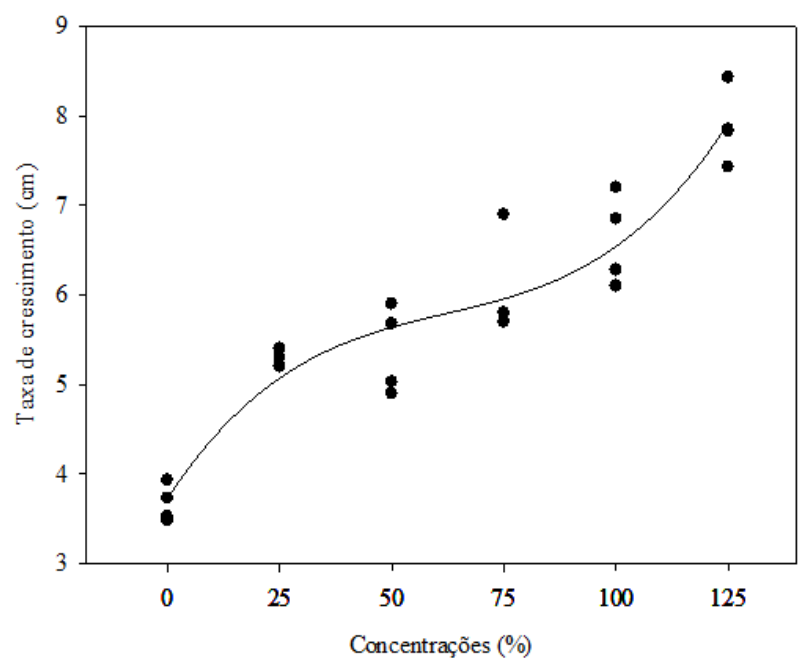

Figura 1. Análise de regressão da variável altura de plantas AP $(\mathrm{cm})$ de mudas de tomate, submetidas dos 5 aos 50 dias após a germinação, a diferentes concentrações de água residuária de piscicultura $\left(\mathrm{R}^{2}=91,40 ; \mathrm{y}=0,1095+0,3451 \mathrm{x}-\right.$ $\left.0,9052 x^{2}+0,8657 x^{3}\right)$. 
A altura de plantas (AP), também apresentou comportamento cúbico de regressão para as concentrações de $0(\mathrm{~F}=$ 143,74; $P=0,00), 25(\mathrm{~F}=88,38 ; \quad P=0,00), 50$ $(\mathrm{F}=90,80 ; \quad P=0,00), 75(\mathrm{~F}=429,18 ; \quad P=$ $0,00), 100(\mathrm{~F}=170,19 ; P=0,00)$ e $125 \%(\mathrm{~F}=$ 327,77; $P=0,00$ ) como mostra a figura 2 . Em ordem decrescente, as concentrações de água residuária que originaram maiores $\mathrm{AP}$ de tomate ao final da avaliação (50 DAG) foram as de $125,25,100,75,50$ e $0 \%$, com valores finais de altura de 10,55; 8,$99 ; 8,73 ; 8,18 ; \quad 7,92$ e $6,56 \mathrm{~cm}$, respectivamente. As plantas do tratamento com $125 \%$ de água se desenvolveram mais rapidamente.
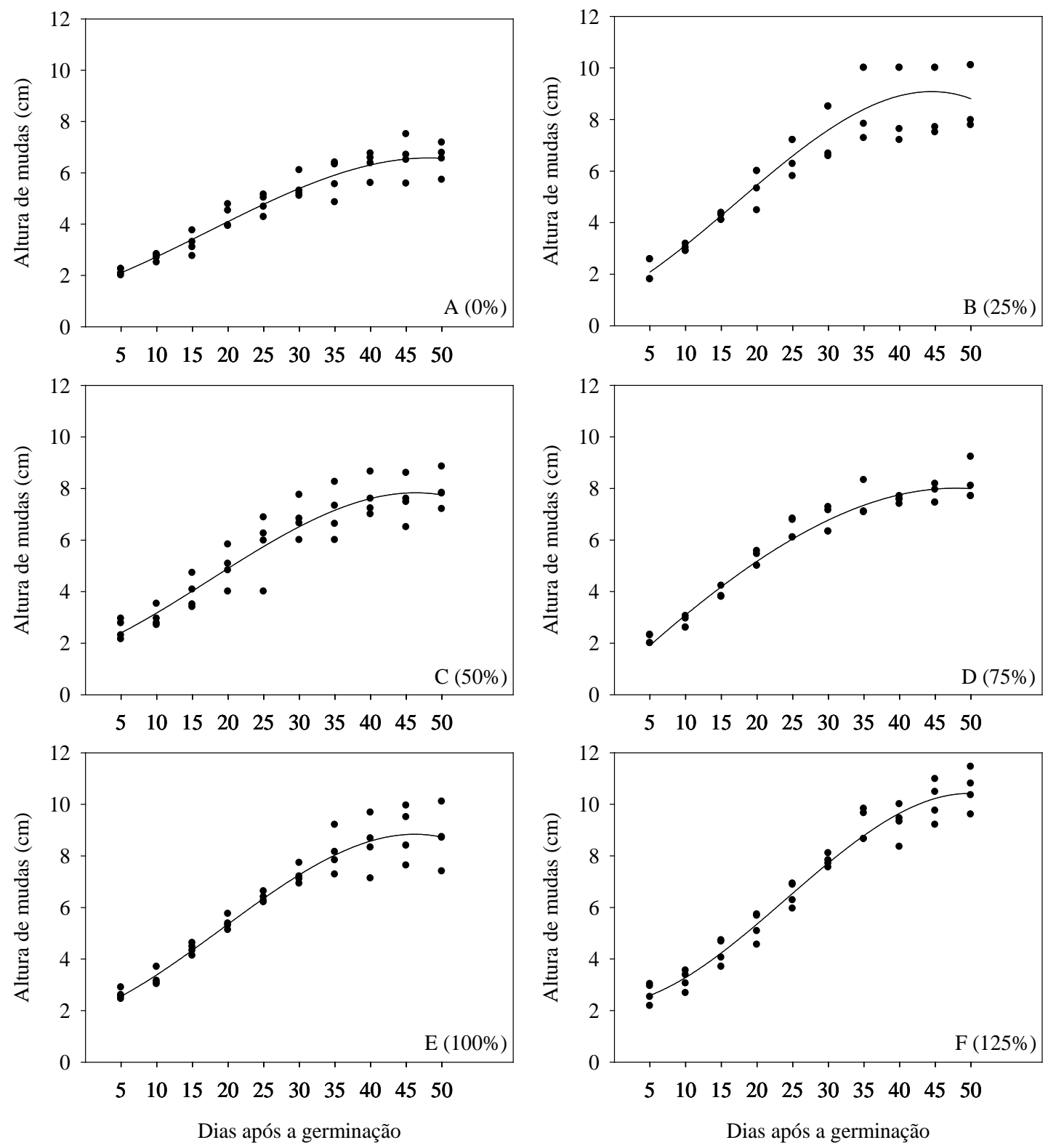

Figura 2. Análise de regressão da variável altura de plantas AP (cm) de mudas de tomate, submetidas dos 5 aos 50 dias após a germinação, a diferentes concentrações de água residuária de piscicultura (Figuras A a F). Fig.2A $\left(R^{2}=82,02 ; \quad y=-0,3523+0,1424 x-0,3902 x^{2}+0,3630 x^{3}\right) . \quad$ Fig.2B $\quad\left(R^{2}=74,39 ; \quad y=-0,3167+0,1354 x-\right.$ 0,3612 $\left.x^{2}+0,3896 x^{3}\right)$. Fig.2C $\left(R^{2}=73,49 ; y=-0,6775+0,2107 x-0,6075 x^{2}+0,5481 x^{3}\right)$. Fig.2D $\left(R^{2}=82,09 ; y=-\right.$ $\left.0,3098+0,1300 x+0,1900 x^{2}\right)$. Fig.2E $\left(R^{2}=87,89 ; y=-0,4758+0,1626 x-0,3644 x^{2}\right)$.Fig.2F $\left(R^{2}=88,14 ; y=-\right.$ $\left.0,1095+0,1013 x-0,1112 x^{2}\right)$. 
Castro et al., (2003) avaliando o uso de efluente de viveiro de peixes e água de poço de irrigação do tomate cereja em diferentes adubos, Observaram o efeito significativo para o tipo de água e a irrigação com efluente provocou um aumento da produtividade, sempre que as necessidades das plantas não eram adequadamente supridas pela adubação.

Na Tabela 2 estão os resultados da variável largura das folhas (LF) das mudas de tomate. Os resultados mostraramse significativamente diferentes aos 5, 10, 25 e 30 DAG dentro das concentrações de água residuária estudadas. A concentração de $125 \%$ originou plantas com maior largura de folhas aos 5 DAG. Esse padrão também foi observado nas concentrações de $25 \%$ aos 10 DAG, $25 \%$ aos 25 DAG e $25 \%$ aos 30 DAG.

Tabela 2. Largura média das folhas ( $\mathrm{cm}$ ) (média $\pm \mathrm{EP}^{1}$ ) de tomate, submetidas a diferentes concentrações de água residuária de piscicultura, até os 50 DAG.

\begin{tabular}{|c|c|c|c|c|c|c|c|c|c|}
\hline \multirow[b]{2}{*}{ (DAG) } & \multicolumn{6}{|c|}{ Concentrações (\%) } & \multirow[b]{2}{*}{$\mathrm{F}$} & \multirow[b]{2}{*}{$P$} & \multirow[b]{2}{*}{$\mathrm{CV}$} \\
\hline & 0 & 25 & 50 & 75 & 100 & 125 & & & \\
\hline \multirow{2}{*}{5} & $0,37 \pm$ & $0,41 \pm$ & $0,36 \pm$ & $0,40 \pm$ & $0,36 \pm$ & $0,46 \pm$ & \multirow{2}{*}{4,46} & \multirow{2}{*}{0,00} & \multirow{2}{*}{8,76} \\
\hline & $0,01 \mathrm{~b}$ & $0,01 \mathrm{ab}$ & $0,03 \mathrm{~b}$ & $0,01 \mathrm{ab}$ & $0,01 \mathrm{~b}$ & $0,01 \mathrm{a}$ & & & \\
\hline \multirow{2}{*}{10} & $0,45 \pm$ & $0,61 \pm$ & $0,45 \pm$ & $0,45 \pm$ & $0,46 \pm$ & $0,49 \pm$ & \multirow{2}{*}{6,96} & \multirow{2}{*}{0,00} & \multirow{2}{*}{10,08} \\
\hline & $0,01 \mathrm{~b}$ & $0,04 \mathrm{a}$ & $0,01 \mathrm{~b}$ & $0,01 \mathrm{~b}$ & $0,00 \mathrm{~b}$ & $0,02 \mathrm{~b}$ & & & \\
\hline \multirow{2}{*}{15} & $1,16 \pm$ & $1,76 \pm$ & $1,65 \pm$ & $1,40 \pm$ & $1,46 \pm$ & $1,39 \pm$ & \multirow{2}{*}{1,69} & \multirow{2}{*}{0,18} & \multirow{2}{*}{22,07} \\
\hline & 0,10 a & $0,30 \mathrm{a}$ & 0,18 a & $0,10 \mathrm{a}$ & $0,04 \mathrm{a}$ & $0,07 \mathrm{a}$ & & & \\
\hline \multirow{2}{*}{20} & $1,26 \pm$ & $1,96 \pm$ & $1,63 \pm$ & $1,73 \pm$ & $1,56 \pm$ & $1,48 \pm$ & \multirow{2}{*}{2,63} & \multirow{2}{*}{0,06} & \multirow{2}{*}{18,00} \\
\hline & 0,08 a & $0,31 \mathrm{a}$ & 0,09 a & 0,05 a & $0,04 \mathrm{a}$ & 0,09 a & & & \\
\hline \multirow{2}{*}{25} & $1,31 \pm$ & $1,75 \pm$ & $1,47 \pm$ & $1,73 \pm$ & $1,61 \pm$ & $1,55 \pm$ & \multirow{2}{*}{3,38} & \multirow{2}{*}{0,02} & \multirow{2}{*}{11,26} \\
\hline & $0,08 \mathrm{~b}$ & $0,14 \mathrm{a}$ & $0,07 \mathrm{~b}$ & $0,01 \mathrm{a}$ & $0,08 a b$ & $0,07 \mathrm{ab}$ & & & \\
\hline \multirow{2}{*}{30} & $1,38 \pm$ & $2,08 \pm$ & $1,70 \pm$ & $1,78 \pm$ & $1,76 \pm$ & $1,82 \pm$ & \multirow{2}{*}{3,65} & \multirow{2}{*}{0,01} & \multirow{2}{*}{13,44} \\
\hline & $0,08 \mathrm{~b}$ & $0,24 \mathrm{a}$ & $0,07 \mathrm{ab}$ & $0,08 a b$ & $0,05 a b$ & $0,04 \mathrm{ab}$ & & & \\
\hline \multirow{2}{*}{35} & $1,43 \pm$ & $1,86 \pm$ & $1,54 \pm$ & $1,76 \pm$ & $1,68 \pm$ & $1,83 \pm$ & \multirow{2}{*}{1,31} & \multirow{2}{*}{0,30} & \multirow{2}{*}{17,43} \\
\hline & 0,13 a & $0,25 \mathrm{a}$ & 0,13 a & $0,14 \mathrm{a}$ & 0,06 a & $0,08 \mathrm{a}$ & & & \\
\hline 40 & $1,44 \pm$ & $1,98 \pm$ & $1,58 \pm$ & $1,87 \pm$ & $1,70 \pm$ & $1,91 \pm$ & 195 & 012 & 1721 \\
\hline 40 & 0,10 a & $0,24 \mathrm{a}$ & $0,12 \mathrm{a}$ & 0,18 a & $0,08 \mathrm{a}$ & $0,10 \mathrm{a}$ & J, & 0,13 & $1 /, 21$ \\
\hline 45 & $1,36 \pm$ & $1,99 \pm$ & $1,53 \pm$ & $1,83 \pm$ & $1,64 \pm$ & $1,79 \pm$ & 275 & 005 & \\
\hline 45 & $0,02 \mathrm{a}$ & $0,29 \mathrm{a}$ & $0,08 \mathrm{a}$ & $0,12 \mathrm{a}$ & $0,00 \mathrm{a}$ & $0,02 \mathrm{a}$ & 2,15 & 0,0 & $0,0<$ \\
\hline 50 & $1,60 \pm$ & $1,97 \pm$ & $1,50 \pm$ & $1,88 \pm$ & $1,45 \pm$ & $1,60 \pm$ & 217 & 0.10 & 7.77 \\
\hline 50 & $0,08 \mathrm{a}$ & $0,30 \mathrm{a}$ & $0,04 \mathrm{a}$ & $0,06 \mathrm{a}$ & $0,05 \mathrm{a}$ & $0,08 \mathrm{a}$ & $2,1 /$ & 0,10 & $1 /, 1 /$ \\
\hline
\end{tabular}

${ }^{1}$ Médias seguidas pela mesma letra, dentro de cada linha, não diferem entre si ao nível de 5\% de probabilidade pelo teste Tukey.

O maior valor comparativo para a variável LF, entre os 60 e 5 DAG, foi observado para a concentração de $25 \%$, com taxa de crescimento em largura de cerca de $79 \%$ a mais aos 50 DAG em comparação com os 5 DAG. Nascimento et al., (2016) analisando a largura média das folhas de pimentão em diferentes concentrações de água residuária de piscicultura, não encontraram diferenças significativas entre as concentrações de água residuária estudadas.
Os resultados da variável número de folhas (NF) sofreu influência do tipo de concentração de água residuária, apenas, aos 5, 10, 25 e 30 DAG, o que pode ser visualizado na Tabela 3. Na concentração da solução fornecida às mudas de tomate de $25 \%$, o número médio de folhas verdadeiras foi maior nos 10, 25 e 30 DAG. Aos 5 DAG, o maior número de folhas verdadeiras das mudas de tomate foram observadas na concentração de $125 \%$. Tal fato deve-se, provavelmente, ao 
IRRIGAÇÃO COM EFLUENTE DE PISCICULTURA NO CULTIVO DE MUDAS DE TOMATE

maior volume de concentração que condições satisfatórias ao desenvolvimento das envolve as raízes das mudas, propiciando mesmas (OLIVEIRA et al. 1993).

Tabela 3. Número de folhas verdadeiras (média $\pm \mathrm{EP}^{1}$ ) de tomate, submetidas a diferentes concentrações de água residuária de piscicultura, até os 50 DAG.

\begin{tabular}{|c|c|c|c|c|c|c|c|c|c|}
\hline \multirow[b]{2}{*}{ (DAG) } & \multicolumn{6}{|c|}{ Concentrações (\%) } & \multirow[b]{2}{*}{$\mathrm{F}$} & \multirow[b]{2}{*}{$P$} & \multirow[b]{2}{*}{$\mathrm{CV}$} \\
\hline & 0 & 25 & 50 & 75 & 100 & 125 & & & \\
\hline 5 & $\begin{array}{l}0,37 \pm \\
0,01 \mathrm{~b}\end{array}$ & $\begin{array}{l}0,41 \pm \\
0,01 \mathrm{ab}\end{array}$ & $\begin{array}{l}0,36 \pm \\
0,03 \mathrm{~b}\end{array}$ & $\begin{array}{c}0,40 \pm \\
0,01 \mathrm{ab}\end{array}$ & $\begin{array}{l}0,36 \pm \\
0,01 \mathrm{~b}\end{array}$ & $\begin{array}{l}0,46 \pm \\
0,01 \mathrm{a}\end{array}$ & 4,46 & 0,00 & 8,76 \\
\hline 10 & $\begin{array}{l}0,45 \pm \\
0,01 \mathrm{~b}\end{array}$ & $\begin{array}{l}0,61 \pm \\
0,04 \mathrm{a}\end{array}$ & $\begin{array}{l}0,45 \pm \\
0,01 \mathrm{~b}\end{array}$ & $\begin{array}{l}0,45 \pm \\
0,01 \mathrm{~b}\end{array}$ & $\begin{array}{l}0,46 \pm \\
0,00 \mathrm{~b}\end{array}$ & $\begin{array}{l}0,49 \pm \\
0,02 \mathrm{~b}\end{array}$ & 6,96 & 0,00 & 10,08 \\
\hline 15 & $\begin{array}{l}1,16 \pm \\
0,10 a\end{array}$ & $\begin{array}{l}1,78 \pm \\
0,30 \mathrm{a}\end{array}$ & $\begin{array}{l}1,65 \pm \\
0,18 \mathrm{a}\end{array}$ & $\begin{array}{l}1,40 \pm \\
0,10 \mathrm{a}\end{array}$ & $\begin{array}{l}1,46 \pm \\
0,04 \mathrm{a}\end{array}$ & $\begin{array}{l}1,39 \pm \\
0,07 \mathrm{a}\end{array}$ & 1,69 & 0,18 & 22,07 \\
\hline 20 & $\begin{array}{l}1,26 \pm \\
0,08 \mathrm{a}\end{array}$ & $\begin{array}{l}1,96 \pm \\
0,31 \mathrm{a}\end{array}$ & $\begin{array}{l}1,63 \pm \\
0,09 \mathrm{a}\end{array}$ & $\begin{array}{l}1,73 \pm \\
0,05 \mathrm{a}\end{array}$ & $\begin{array}{l}1,56 \pm \\
0,04 \mathrm{a}\end{array}$ & $\begin{array}{l}1,48 \pm \\
0,09 \mathrm{a}\end{array}$ & 2,63 & 0,06 & 18,00 \\
\hline 25 & $\begin{array}{l}1,31 \pm \\
0,08 \mathrm{~b}\end{array}$ & $\begin{array}{c}1,75 \pm \\
014 \mathrm{a}\end{array}$ & $\begin{array}{l}1,47 \pm \\
0,07 \mathrm{ab}\end{array}$ & $\begin{array}{l}1,73 \pm \\
0,01 \mathrm{a}\end{array}$ & $\begin{array}{l}1,61 \pm \\
0,08 \mathrm{ab}\end{array}$ & $\begin{array}{c}1,55 \pm \\
0,07 \mathrm{ab}\end{array}$ & 3,38 & 0,02 & 11,27 \\
\hline 30 & $\begin{array}{l}1,38 \pm \\
0,08 \mathrm{~b}\end{array}$ & $\begin{array}{l}2,08 \pm \\
0,24 \mathrm{a}\end{array}$ & $\begin{array}{l}1,70 \pm \\
0,07 \mathrm{ab}\end{array}$ & $\begin{array}{c}1,78 \pm \\
0,08 \mathrm{ab}\end{array}$ & $\begin{array}{l}1,76 \pm \\
0,05 \mathrm{ab}\end{array}$ & $\begin{array}{c}1,82 \pm \\
0,04 \mathrm{ab}\end{array}$ & 3,65 & 0,01 & 13,44 \\
\hline 35 & $\begin{array}{l}1,43 \pm \\
0,13 \mathrm{a}\end{array}$ & $\begin{array}{l}1,86 \pm \\
0,25 \mathrm{a}\end{array}$ & $\begin{array}{l}1,54 \pm \\
0,13 \mathrm{a}\end{array}$ & $\begin{array}{l}1,76 \pm \\
0,14 \mathrm{a}\end{array}$ & $\begin{array}{l}1,68 \pm \\
0,06 \mathrm{a}\end{array}$ & $\begin{array}{l}1,83 \pm \\
0,08 \mathrm{a}\end{array}$ & 1,31 & 0,30 & 17,43 \\
\hline 40 & $\begin{array}{l}1,44 \pm \\
0,10 \mathrm{a}\end{array}$ & $\begin{array}{l}1,98 \pm \\
0,24 \mathrm{a}\end{array}$ & $\begin{array}{l}1,58 \pm \\
0,12 \mathrm{a}\end{array}$ & $\begin{array}{c}1,87 \pm \\
018 \mathrm{a}\end{array}$ & $\begin{array}{l}1,70 \pm \\
0,08 \mathrm{a}\end{array}$ & $\begin{array}{l}1,91 \pm \\
0,10 \mathrm{a}\end{array}$ & 1,95 & 0,13 & 17,21 \\
\hline 45 & $\begin{array}{l}1,36 \pm \\
0,02 \mathrm{a}\end{array}$ & $\begin{array}{l}1,99 \pm \\
0,29 \mathrm{a}\end{array}$ & $\begin{array}{l}1,53 \pm \\
0,08 \mathrm{a}\end{array}$ & $\begin{array}{l}1,83 \pm \\
0,12 \mathrm{a}\end{array}$ & $\begin{array}{l}1,64 \pm \\
0,00 \mathrm{a}\end{array}$ & $\begin{array}{l}1,79 \pm \\
0,02 \mathrm{a}\end{array}$ & 2,75 & 0,05 & 16,02 \\
\hline 50 & $\begin{array}{l}1,60 \pm \\
0,08 \mathrm{a}\end{array}$ & $\begin{array}{l}1,97 \pm \\
0,30 \mathrm{a}\end{array}$ & $\begin{array}{l}1,50 \pm \\
0,04 \mathrm{a}\end{array}$ & $\begin{array}{l}1,38 \pm \\
0,06 \mathrm{a}\end{array}$ & $\begin{array}{l}1,45 \pm \\
0,05 \mathrm{a}\end{array}$ & $\begin{array}{l}1,60 \pm \\
0,08 \mathrm{a}\end{array}$ & 2,17 & 0,10 & 17,77 \\
\hline
\end{tabular}

${ }^{1}$ Médias seguidas pela mesma letra, dentro de cada linha, não diferem entre si ao nível de 5\% de probabilidade pelo teste Tukey.

Os resultados das variáveis peso de matéria fresca do sistema radicular (PMFSR), peso de matéria fresca da parte aérea (PMFPA), e peso de matéria seca do sistema radicular (PMSSR) e peso de matéria seca da parte aérea (PMSPA) são apresentados na Tabela 4.

Tabela 4. Peso de matéria fresca do sistema radicular (PMFSR), peso de matéria fresca da parte aérea (PMFPA), peso de matéria seca do sistema radicular (PMSSR) e peso de matéria seca da parte aérea (PMSPA) em g, (média \pm EP1) de mudas de pimentão, submetidas a diferentes concentrações de água residuária de piscicultura, até os 50 DAG.

\begin{tabular}{|c|c|c|c|c|}
\hline Conc. (\%) & PMFSR & PMFPA & PMSSR & PMSPA \\
\hline 0 & $1,12 \pm 0,042 \mathrm{a}$ & $0,382 \pm 0,046 \mathrm{~b}$ & $0,055 \pm 0,001 \mathrm{~b}$ & $0,040 \pm 0,006 \mathrm{~b}$ \\
\hline 25 & $1,293 \pm 0,208 \mathrm{a}$ & $0,555 \pm 0,124 \mathrm{ab}$ & $0,173 \pm 0,059 \mathrm{a}$ & $0,072 \pm 0,016 a b$ \\
\hline 50 & $1,102 \pm 0,067 \mathrm{a}$ & $0,382 \pm 0,087 \mathrm{~b}$ & $0,072 \pm 0,002 \mathrm{ab}$ & $0,054 \pm 0,011 b$ \\
\hline 75 & $0,812 \pm 0,063 \mathrm{a}$ & $0,277 \pm 0,035 b$ & $0,062 \pm 0,006 \mathrm{~b}$ & $0,046 \pm 0,006 b$ \\
\hline 100 & $1,105 \pm 0,048 \mathrm{a}$ & $0,478 \pm 0,049 a b$ & $0,078 \pm 0,003 \mathrm{ab}$ & $0,072 \pm 0,008 \mathrm{ab}$ \\
\hline 125 & $1,200 \pm 0,131 \mathrm{a}$ & $0,780 \pm 0,102 \mathrm{a}$ & $0,107 \pm 0,013 \mathrm{ab}$ & $0,112 \pm 0,011 \mathrm{a}$ \\
\hline $\mathrm{F}$ & 2,12 & 4,61 & 3,05 & 6,05 \\
\hline$P$ & 0,07 & 0,00 & 0,01 & 0,00 \\
\hline $\mathrm{CV}$ & 31,66 & 54,62 & 86,73 & 51,22 \\
\hline
\end{tabular}

seguidas pela mesma letra, dentro de cada coluna, não diferem entre si ao nível de 5\% de probabilidade pelo teste Tukey. 
As variáveis PMFSR, PMFPA e PMSPA obtiveram maiores valores na concentração de $125 \%$ em comparação com as demais. Para o parâmetro PMFSR das mudas de tomateiro, não houve diferença significativa entre as concentrações avaliadas. Os resultados da variável PMSSR foram maiores na concentração de $25 \%$.

As mudas irrigadas com efluente de piscicultura, possivelmente devido ao fato deste ser rico em matéria orgânica e mineral, melhoraram seu desenvolvimento, independente do volume de concentrações presente nas células das bandejas. Segundo Nunes et al. (2002) os fertilizantes utilizados no cultivo de organismos aquáticos possuem a capacidade de aumentar as concentrações de nitrogênio e fósforo na água, o que pode ter promovido uma fertilização das mudas de tomate.

Freitas et al. (2008) comparando o efeito de irrigação utilizando água de poço com a utilização de efluente de piscicultura na produção de mudas de pimentão, não observaram diferenças significativas para massa seca do caule, massa seca da raiz, massa seca da folha, massa fresca da raiz número de folhas e tamanho da raiz.

A irrigação realizada com água residuária de piscicultura favoreceram o desenvolvimento de todas as plantas, com uma produção semanal considerável por metro quadrado, em um cultivo de aproximadamente dois meses, o que para um sistema de produção similar garante uma renda constante durante todo o período de safra do tomate cereja, além do ganho com a comercialização dos peixes.

\section{CONCLUSÕES}

Os tratamentos com diferentes concentrações de água residuária de piscicultura propiciaram diferenças significativas nas variáveis, largura das folhas e altura das plantas de tomate. sendo que quanto maior a concentração maior foram os valores dessas variáveis, devido a maior disponibilidade de nutrientes a planta.

A água residuária da piscicultura apresenta-se como alternativa para reuso do ponto de vista nutricional para as plantas, diminuindo o impacto ambiental dos efluentes desse tipo de criação animal.

\section{REFERÊNCIAS}

AUGUSTO, D. C. C.; GUERRINI, I. A.; ENGEL, V. L.; ROUSSEAU, G. X. Utilização de esgotos domésticos tratados através de um sistema biológico na produção de mudas de Croton floribundus spreng (Capixigui) e Capaifera lagndorffi desf (Capoíba). Revista Árvore, Viçosa, v.27, n.3, p.335-342, 2003.

CASTRO, R. S.; AZEVEDO, C.B.; BEZERRA NETO, F.; TORQUATO, J. E. Produtividade do tomate cereja cultivado em sistema orgânico, irrigado com efluente de piscicultura. Horticultura Brasileira, v. 21, n.2, 2003.

FREITAS, A. V. L.; MEDEIROS, M. A.; GUIMARÃES, I. P.; MADALENA, J. A. S.; Maracajá, P. B. Produção de mudas de pimentão em função do tipo de bandeja e da água de irrigação. Revista Verde, v.3, n.3. p.106-109, 2008.

GOMES, M. A. F. Água: sem ela seremos o planeta Marte de amanhã. In: CONGRESSO BRASILEIRO DE ENGENHARIA SANITÁRIA E AMBIENTAL, Belo Horizonte, Anais... Belo Horizonte, 2007.

HUSSAR, G. J.; PARADELA, A. L.; BASTOS, M. C.; REIS, T. K. B.; JONAS, T. C.; SERRA, W.; GOMES, J. P. Efeitos do uso do efluente de reator anaeróbio compartimentado na fertirrigação da beterraba. Revista Engenharia Ambiental, v.2, n.1, p.35-45, 2005.

KUMMER, A. C. B.; SILVA, I. P. F.; LOBO, T. F.; GRASSI FILHO, H. Qualidade da agua residuaria para irrigação do trigo. In: IV WORKSHOP INTERNACIONAL DE INOVAÇÕES TECNOLÓGICAS NA IRRIGAÇÃO E I INOVAGRI INTERNATIONAL MEETING, Fortaleza, Anais... Fortaleza, 2012. 
LUCHESE, A. V.; JUNIOR, A. C. G.; LUCHESE, E. B.; BRACCINI, M. do C. L. Emergência e absorção de cobre por plantas de milho (Zea mays) em resposta ao tratamento de sementes com cobre. Ciência Rural, v.34, n.6, p.1949-1952, 2004.

MEDEIROS, S. S.; SOARES, F. A. L.; GHEYI, H. R.; FERNANDES, P. D. Uso de água residuária de origem urbana no cultivo de gérberas: efeito nos componentes de produção. Engenharia Agrícola, v. 27, n.2, p.569-578, 2007.

MEHNERT, D. U. Reuso de efluente doméstico na agricultura e a contaminação ambiental por vírus entéricos humanos. Biológico, São Paulo, v.65, n.1/2, p.19-21, 2003.

NASCIMENO, T. S.; FLORIANO, L. S.; PEREIRA, A. I. A.; MONTEIRO, R. N. F.; SALES, M. A. L. Produção de mudas de pimentão irrigadas com efluentes de piscicultura. Revista Brasileira de Agricultura Irrigada. v.10, nº 1, p. 449 - 459, 2016.

NUNES, A. J. P. Tratamentos de efluentes e recirculação de água na engorda de camarão marinho. Revista Panorâmica da Aqüicultura. Rio de Janeiro, vol.12, nº71, p. 27-39, 2002.

OLIVEIRA, R. P.; SCIVITTARO, W. B.; VASCONCELLOS, L. A. B. C. Avaliação de mudas de maracujazeiro em função do substrato e do tipo de bandeja. Scientia Agrícola, Piracicaba, v.50, n.2, p.261-266, 1993.

RIBEIRO, I. A. V.; TERESO, M. J. A.; ABRAHÃO, R. F. Análise ergonômica do trabalho em unidades de beneficiamento de tomates de mesa: movimentação manual de cargas. Ciência Rural, v.39, n.4, p.1083-1089, 2009.
SALES, M. A. L. Balanço hídrico na subbacia hidrográfica do Boi Branco - SP para gestão sustentável da agricultura irrigada. 2014. 120 f. Dissertação (Mestrado em Agronomia - Irrigação e Drenagem) - Faculdade de Ciências Agronômicas, Universidade Estadual Paulista “Júlio de Mesquita Filho”.

SANCHES, A.; MONTEGGIA, L. O.; GONÇALVES, H. R.; PADILHA, R. dos S. Utilização de efluente de reator UASB e lagoas de estabilização na fertirrigação do milho como alternativa de uso. In: CONGRESSO BRASILEIRO DE ENGENHARIA SANITÁRIA E AMBIENTAL, Belo Horizonte, Anais... Belo Horizonte, 2007.

SANDRI, D. Irrigação da cultura da alface com água residuária tratada com leitos cultivados com macrófita. 2003. 186 f. Tese (Doutorado em Engenharia de Água e Solo) Faculdade de Engenharia Agrícola, Universidade Estadual de Campinas.

SANDRI, D.; MATSURA, E. E.; TESTEZLAF, R. Desenvolvimento da alface Elisa em diferentes sistemas de irrigação com água residuária. Revista Brasileira de Engenharia Agrícola e Ambiental, v.11, n.1, p.17-29, 2007.

SARIG, S. The immigration for fish culture into general farm irrigation systems in Israel. The Israeli Journal of AquacultureBamidgeh. v.1, n.36, p.16-20. 1994.

SOUSA, J. T.; CEBALLOS, B. S. O.; HENRIQUE, I. N.; DANTAS, P. J.; LIMA, S. M. S. Reuso de água residuária na produção de pimentão (Capsicum annuum L.). Revista Brasileira de Engenharia Agrícola e Ambiental, v.10, n.1, p.89-96, 2006. 